\title{
Alveolar recruitment in acute respiratory distress syndrome: should we open the lung (no matter what) or may accept (part of) the lung closed?
}

\author{
Lorenzo Del Sorbo ${ }^{1}$, Tommaso Tonetti ${ }^{2,3}$ and V. Marco Ranieri ${ }^{4^{*}}$ (I)
}

(C) 2019 Springer-Verlag GmbH Germany, part of Springer Nature

The conceptual model of acute respiratory distress syndrome (ARDS) includes: (a) lung inflammation; (b) severe hypoxemia; (c) edema, hyaline membranes, and alveolar hemorrhage. Common denominator is the loss of aerated lung tissue due to alveolar collapse. To open collapsed alveoli has been, therefore, proposed to restore gas exchange [1] with interventions such as positive endexpiratory pressure (PEEP), recruitment maneuvers, and prone position [2]. Moreover, loss of lung volume may contribute to ventilator-induced lung injury (VILI) [2]. We will review the physiology of alveolar recruitment and address the implications of the "full recruitment" vs "partial recruitment" approaches (Fig. 1).

\section{The physiology of alveolar recruitment}

Although the mechanisms and the consequences of alveolar recruitment had been thoroughly studied, it is only after the late 80s [3] and early 90s [4] that alveolar recruitment was quantified. Gattinoni et al. used quantitative analysis of lung CT-scan and showed that PEEPinduced increase in oxygenation was strongly related to a progressive increase in aerated lung tissue, and, thus,

\footnotetext{
*Correspondence: m.ranieri@unibo.it

${ }^{4}$ Anesthesia and Intensive Care Medicine, Alma Mater Studiorum University of Bologna, Policlinico di Sant'Orsola, Via Massarenti 9, 40138 Bologna, Italy

Full author information is available at the end of the article
}

Lorenzo Del Sorbo and Tommaso Tonetti equally contributed to the article and should both be considered as "First author".

Supported by Ministero dell'Università e della Ricerca (2017J4BE7A) PRIN 2017. to the recruitment of perfused and collapsed lung units [3]. Ranieri et al. used quantitative analysis of the volume-pressure curve at different PEEP levels and showed that in some patients, PEEP shifted the curve upward along the volume axis indicating an increase in volume for the same pressure (recruitment). In other patients, PEEP shifted the curve along the pressure axis indicating an increase in pressure for the same volume (hyperinflation) (Fig. 2) [4]. Later, Gattinoni et al. confirmed that the amount of potentially recruited lung was highly variable and, although the study was performed in 68 patients only, it showed that patients with a larger amount of potentially recruitable alveoli tended to have a higher mortality than patients who had smaller amount of alveoli potentially recruitable [5].

The effects of alveolar recruitment on gas exchange are mainly due to recruitment-induced modifications in venous admixture $\left(Q_{s} / Q_{t}\right)$ and dead space. An increase in $\mathrm{PaO}_{2}$ is normally observed after applying PEEP, and this increase is highly correlated with a decrease in $Q_{s} / Q_{t}$ [6]. Interestingly, the increase in $\mathrm{PaO}_{2}$ is significant also in the non-recruitable patients, in whom the decrease in $Q_{s} / Q_{t}$ cannot be solely explained by re-aeration of alveoli with a low VA/Q. Thus, the effect of PEEP on oxygenation may be-at least in part-explained by a reduction in $Q_{s} / Q_{t}$ due to a PEEP-related decrease in cardiac output [6].

$\mathrm{PaCO}_{2}$ is mainly dependent on dead space $\left(V_{\mathrm{D}} / V_{\mathrm{T}}\right)$. Lung volume has an ambiguous, "U" shaped, effect on $V_{\mathrm{D}} / V_{\mathrm{T}}$, depending mostly on the recruitment response to the applied PEEP levels [7]. Indeed, opening of previously closed lung units may reduce $V_{\mathrm{D}} / V_{\mathrm{T}}$ and improve $\mathrm{CO}_{2}$

\section{国




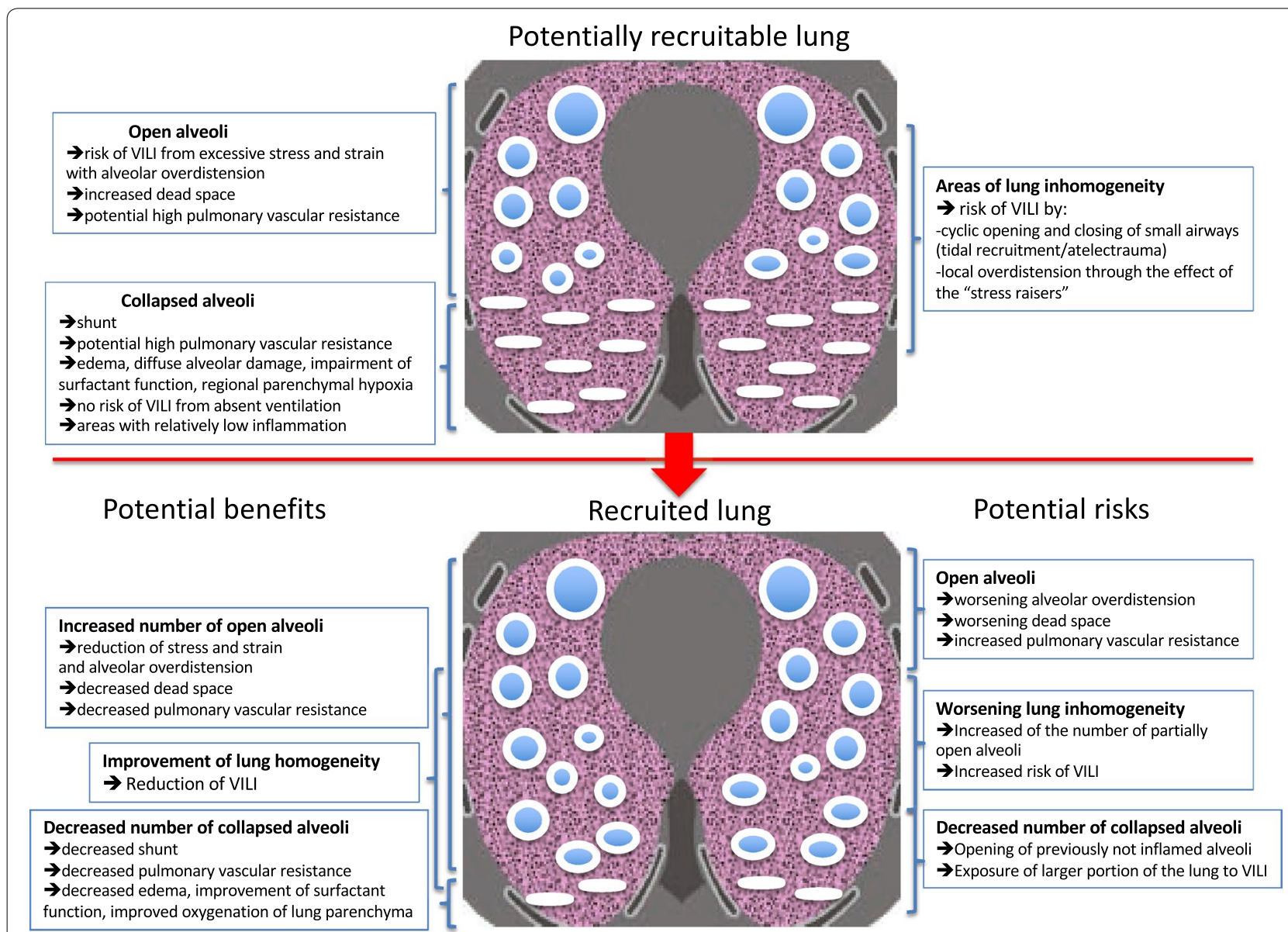

Fig. 1 Physiological implications of alveolar recruitment

elimination. However, if the increase in aerated units is accompanied by an even higher increase in overinflated units, this leads invariably to an increase in $V_{\mathrm{D}} / V_{\mathrm{T}}$ and in $\mathrm{PaCO}_{2}[7]$.

\section{Alveolar recruitment and VILI}

Overall, the above presented data would suggest that recruitment of collapsed alveolar units should be maximized in patients with high potential for recruitment to improve their outcome [1]. Nevertheless, evidences failed to conclusively demonstrate that strategies leading to "full recruitment" improve outcome, and application of these strategies is prudently suggested (and not recommended) by recent guidelines [8]. Two recent randomized clinical trials showed that (a) maximization of lung recruitment through the use of high-frequency oscillatory ventilation was associated with higher mortality [9]; (b) mechanical ventilation with higher PEEP titrated on best lung compliance had higher mortality compared with patients treated with lower PEEP [10]. We must underline that (a) high-frequency oscillatory ventilation substantially differs from conventional mechanical ventilation; (b) these studies used completely different ventilatory approaches that were applied regardless of individual assessment of patient physiology. Nevertheless, these results seem to point out that maximizing recruitment may not be always the right strategy to minimize alveolar overdistension despite the delivery of low tidal volumes since "high potential for recruitment" [5] may in fact be just a marker of ARDS severity, rather than a pathological feature that need to be reversed. In addition, the change in lung inhomogeneity with mechanical ventilation may be unpredictable [11], and its magnitude may critically modify the relationship between total lung volume and tidal volume.

Lung collapse is not always detrimental. During fetal development, the lung remains collapsed and yet healthy, with gas exchange provided by the placenta. Moreover, the collapsed and infiltrated lung in patients with lobar pneumonia that are not mechanically ventilated can completely heal without any artificial lung recruitment 

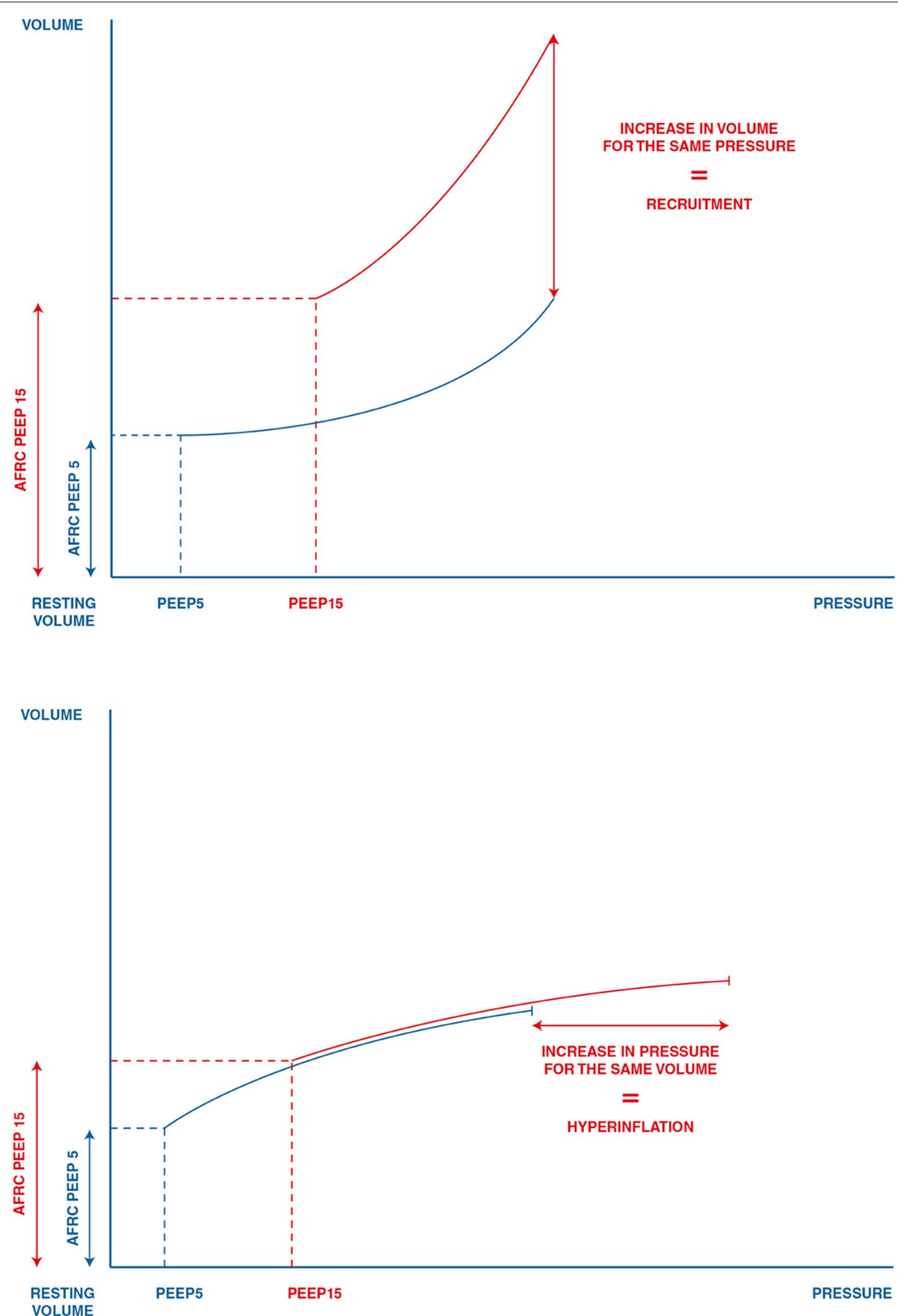

Fig. 2 Quantitative analysis of the volume-pressure curve of the respiratory system to identify and quantify recruitment with PEEP. Top: alveolar recruitment; PEEP shifted upward the curve showing an increase in volume for the same pressure. Bottom: alveolar hyperinflation; PEEP shifted the curve along the pressure axis causing an increase in pressure for almost the same volume. PEEP is positive end-expiratory pressure; resting volume is the volume at the elastic equilibrium point of the respiratory system. $\triangle \mathrm{FRC}$ is the change in functional residual capacity caused by different levels of PEEP. Modified from Reference 4 
maneuver. Furthermore, the first successful treatment provided to patients with pulmonary infection caused by Mycobacterium tuberculosis was the induction of therapeutic pneumothorax with consequent lung collapse [12]. Although fetal development, lobar pneumonia, and M. tuberculosis infection have nothing to do with ARDS, and to date, no clinical trial has irrevocably shown that a "partial recruitment" strategy would result in a better outcome, it should be noted that recent data suggest that the "partial recruitment" approach may better protect the lung from VILI compared to "full recruitment" approaches. Bellani et al. showed in patients with ARDS that aerated pulmonary regions are more inflamed than the collapsed alveolar units [13]. In an ex vivo models, Chu et al. showed that atelectatic lungs had less inflammatory cytokines compared to recruited lungs [14] and Fanelli et al. showed that a "partial recruitment" strategy significantly reduced apoptosis when compared to a "full recruitment" strategy [15].

In conclusion, it remains unknown whether achieving and then maintaining maximal alveolar recruitment are required to minimize lung injury and facilitate lung healing. The clarification of important issues in the pathophysiology of ARDS, such as the interaction between tidal volume and total lung volume (drastically affected by potential for recruitment) and the optimal compromise between alveolar recruitment and overdistension, will guide the design of the future successful clinical trials to improve outcome in patients with ARDS.

\section{Author details \\ ${ }^{1}$ Interdepartmental Division of Critical Care Medicine, University Health Net- work, University of Toronto, Toronto, Canada. ${ }^{2}$ Department of Anesthesiology, Emergency and Intensive Care Medicine, University of Göttingen, Göttingen, Germany. ${ }^{3}$ Department of Anesthesia and Intensive Care, Parma University Hospital, Parma, Italy. ${ }^{4}$ Anesthesia and Intensive Care Medicine, Alma Mater Studiorum University of Bologna, Policlinico di Sant'Orsola, Via Massarenti 9 , 40138 Bologna, Italy.}

\section{Acknowledgements}

The funding has been received from Ministero dell'Università e della Ricerca with Grant No. (2017J4BE7A) PRIN 2017.

\section{Compliance with ethical standards}

\section{Conflicts of interest}

Authors declare no conflict of interests.

\section{Publisher's Note}

Springer Nature remains neutral with regard to jurisdictional claims in published maps and institutional affiliations.

Received: 24 May 2019 Accepted: 6 August 2019

Published online: 16 August 2019

\section{References}

1. Lachmann B (1992) Open up the lung and keep the lung open. Intensive Care Med 18:319-321

2. Slutsky AS, Ranieri VM (2013) Ventilator-induced lung injury. N Engl J Med 369:2126-2136

3. Gattinoni L, Pesenti A, Bombino M et al (1988) Relationships between lung computed tomographic density, gas exchange, and PEEP in acute respiratory failure. Anesthesiology 69:824-832

4. Ranieri VM, Eissa NT, Corbeil C et al (1991) Effects of positive end-expiratory pressure on alveolar recruitment and gas exchange in patients with the adult respiratory distress syndrome. Am Rev Respir Dis 144:544-551

5. Gattinoni L, Caironi P, Cressoni M et al (2006) Lung recruitment in patients with the acute respiratory distress syndrome. N Engl J Med 354:1775-1786

6. Dantzker DR, Lynch JP, Weg JG (1980) Depression of cardiac output is a mechanism of shunt reduction in the therapy of acute respiratory failure. Chest 77:636-642

7. Fengmei G, Jin C, Songqiao L et al (2012) Dead space fraction changes during PEEP titration following lung recruitment in patients with ARDS. Respir Care 57:1578-1585

8. Fan E, Del Sorbo L, Goligher EC et al (2017) An Official American Thoracic Society/European Society of Intensive Care Medicine/Society of Critical Care Medicine Clinical Practice Guideline: mechanical ventilation in adult patients with acute respiratory distress syndrome. Am J Respir Crit Care Med 195:1253-1263

9. Ferguson ND, Cook DJ, Guyatt GH et al (2013) High-frequency oscillation in early acute respiratory distress syndrome. N Engl J Med 368:795-805

10. Cavalcanti AB, Suzumura ÉA, Laranjeira LN et al (2017) Effect of lung recruitment and titrated positive end-expiratory pressure (PEEP) vs low PEEP on mortality in patients with acute respiratory distress syndrome. JAMA 318:1335-1345

11. Terragni PP, Rosboch G, Tealdi A et al (2007) Tidal hyperinflation during low tidal volume ventilation in acute respiratory distress syndrome. Am J Respir Crit Care Med 175:160-166

12. Sakula A (1983) Carlo Forlanini, inventor of artificial pneumothorax for treatment of pulmonary tuberculosis. Thorax 38:326-332

13. Bellani G, Messa C, Guerra L et al (2009) Lungs of patients with acute respiratory distress syndrome show diffuse inflammation in normally aerated regions: a [18F]-fluoro-2-deoxy-D-glucose PET/CT study. Crit Care Med 37:2216-2222

14. Chu EK, Whitehead T, Slutsky AS (2004) Effects of cyclic opening and closing at low- and high-volume ventilation on bronchoalveolar lavage cytokines. Crit Care Med 32:168-174

15. Fanelli V, Mascia L, Puntorieri V et al (2009) Pulmonary atelectasis during low stretch ventilation: "open lung"versus "lung rest" strategy. Crit Care Med 37:1046-1053 